\title{
CDX2 Enhances HTR-8/SVneo Trophoblast Cell Invasion by Altering the Expression of Matrix Metalloproteinases
}

\author{
Rui-Zhe Jia ${ }^{a}$ Gui-Chun Ding ${ }^{b}$ Chun-Mei Gu ${ }^{b}$ Tao Huang ${ }^{b}$ Can Ruia,b \\ Yi-Xiong Wang ${ }^{\mathrm{b}}$ Qin Lu
}

aDepartment of Obstetrics, Nanjing Maternal and Child Health Hospital, Affiliated with Nanjing Medical University, Nanjing, 'Department of Obstetrics and Gynecology, Yangzhou Maternal and Child Health Hospital, Affiliated with Yangzhou Medical University, Yangzhou, 'Department of Obstetrics and Gynecology, Shanghai Jiao Tong University Affiliated Tongren Hospital, Shanghai, China

\section{Key Words}

CDX2 • HTR-8/SVneo cell line • Trophoblast invasion • PI3K/Akt

\begin{abstract}
Background/Aims: The invasion of trophoblast cells into the maternal uterine decidua is critical for normal placentation, establishment of pregnancy and maintenance of fetal growth in humans. Several growth factors and cytokines have been implicated in trophoblast invasion, but the underlying regulatory mechanisms of invasion are not fully understood. Our earlier studies have found that caudal-related homeobox transcription factor 2 (CDX2) is hypomethylated in human pre-eclampsia placental tissues. However, whether CDX2 is involved in trophoblast invasion was unclear. Methods and Results: In this study, we investigated CDX2 function using a human HTR-8/SVneo cell line that overexpressed CDX2. Cell invasion assays demonstrated that CDX2 enhanced trophoblast cell invasiveness. Meanwhile, MTT assays revealed that CDX2 did not affect cell proliferation. Western blot analysis and quantitative real-time PCR demonstrated that the expression level of matrix metalloproteinase-9 (MMP-9) was significantly increased, whereas the expression of tissue inhibitor of metalloproteinase-1 (TIMP-1) was markedly suppressed in the CDX2-overexpressing trophoblast cells. The phosphoinositide-3-kinase (PI3K)/Akt signaling pathway is involved in proliferation, migration, metastasis and invasion. Our study showed that inhibition of PI3K/Akt signaling led to decreased expression of CDX2. Conclusion: We concluded that CDX2 is likely regulated by the PI3K/Akt signaling pathway during trophoblast cell invasion. Our findings may reveal new insights into the regulatory mechanisms of trophoblast cell invasion and may be an important contributor to the pathogenesis of pregnancy-related diseases.
\end{abstract}

R.-Z. Jia and G.-C. Ding contributed equally to this work.

Qin Lu

and Yi-Xiong Wang
Department of Obstetrics and Gynecology, Shanghai Jiao Tong University Affiliated Tongren Hospital, Shanghai 200336 (China)

and Department of Obstetrics and Gynecology, Yangzhou Maternal and Child Health Hospital Affiliated with Yangzhou Medical University, Yangzhou 225002 (China)

E-Mail cuixianwei198615@163.com and E-Mail sbyywyx@163.com 


\section{Introduction}

Trophoblast cells are the main component of the placenta. Normal placental development is characterized by extravillous trophoblast cells penetrating into the maternal decidua and myometrium [1]. Invading trophoblast cells alter the vasculature inside the uterus and partially replace the endothelial cells of the uterine spiral arteries. These activities of trophoblast cells are key events during the establishment, maintenance and termination of physiological pregnancy [2, 3]. Invasiveness is a characteristic of trophoblast cells; however, trophoblast invasion is a strictly controlled physiological event. Any changes in the trophoblast invasion capacity can seriously affect placental function and can threaten maternal and child health. Defective trophoblast invasion may contribute to many pregnancyrelated diseases [4]. For example, excessive invasion can lead to gestational trophoblastic diseases [5], whereas inadequate invasion, specifically restricted endovascular invasion, may cause miscarriage [6], pre-eclampsia [7], fetal growth restriction [8] and other pathological events during pregnancy [9].

Multiple factors including growth factors, cytokines and chemokines are involved in the regulation of trophoblast invasion, such as corin [10], E-cadherin [11], tumor necrosis factor-alpha $(\mathrm{TNF} \alpha)$ [12], matrix metalloproteinase (MMP)-2/MMP-9 [13], interleukin1-beta (IL-1 $\beta$ )/transforming growth factor-beta 1 (TGF- $\beta 1$ ) [14] and tissue inhibitor of metalloproteinase-1 (TIMP-1) [15]. Through the expression and secretion of MMPs, trophoblast cells gain the capacity to degrade extracellular matrix. As a key enzyme during trophoblast invasion, MMP-9 is a member of the MMP family that can efficiently degrade type IV collagen, a main component of the basement membrane [13]. TIMPs are specific inhibitors of MMP-9 that act by forming complexes with MMP-9 $[13,15]$. MMP-9 and TIMP-1 are both closely associated with trophoblast invasion. Down-regulation of MMP-9 and upregulation of TIMP-1 may both lead to decreased trophoblast invasion [16-18].

Caudal-related homeobox transcription factor 2 (CDX2) is a member of the caudalrelated homeobox gene family, with multiple functions in mammalian development, such as proliferation and differentiation [19-22]. Our previous study found that CDX2 is hypomethylated in human severe pre-eclampsia placenta [23]. Additionally, CDX2 is a transcriptional regulator essential for the trophoblast lineage [24, 25]. However, the specific role CDX2 plays in trophoblast invasion is still poorly understood. In this study, we hypothesized that CDX2 is a regulator of trophoblast invasion and that abnormal CDX2 expression may be associated with the pathogenesis of pregnancy-related diseases.

To test our hypothesis, we used the HTR-8/SVneo trophoblast cell line to investigate CDX2 function. By using MTT assay and cell invasion assay, the proliferation and invasive capacity of the HTR-8/SVneo cells were investigated. We detected the expressions of MMP9 and TIMP-1 by Western blot analysis and quantitative real-time PCR. Meanwhile,we evaluated PI3K/AKT signaling in trophoblast cells. Our findings indicate that CDX2 regulates the invasive capacity of the HTR-8/SVneo trophoblasts by altering the expression of MMPs.

\section{Materials and Methods}

\section{Reagents and antibodies}

Primary polyclonal antibodies against CDX2 (Abcam, ab76541, USA), MMP-9 (Abcam, ab38898, USA), TIMP-1 (Cell Signaling, \#8946, USA), PI3K (Abcam, ab22653, USA), and AKT1 (phospho S473, Abcam, ab66138) were used at 1:1000 dilution. Rabbit polyclonal anti- $\beta$-actin antibody (Beyotime, AA128) was used at 1:1,000 dilution (final concentration $1 \mu \mathrm{g} / \mathrm{ml}$ ) as a loading control for western blotting. Horseradish peroxidase (HRP)-conjugated goat anti-rabbit IgG (Abcam, ab6721, USA) was used at 1:5,000 dilution (final concentration $0.2 \mu \mathrm{g} / \mathrm{ml}$ ) as the secondary antibody. 
Jia et al.: CDX2 Enhances HTR-8/SVneo Cell Invasion through MMP Pathway

Cell culture and transfection

The HTR-8/SVneo cells (purchased from the Cell Bank of the Chinese Academy of Sciences, China) were maintained in RPMI-1640 medium (Invitrogen, USA) supplemented with 10\% fetal bovine serum (FBS), $100 \mu \mathrm{g} / \mathrm{mL}$ streptomycin and $100 \mathrm{U} / \mathrm{mL}$ penicillin under standard culture conditions $\left(37^{\circ} \mathrm{C}\right.$ and $5 \%$ CO2 incubator) [26]. The CDX2 expression plasmid was constructed by inserting the entire coding region of the human CDX2 cDNA (synthesized by Shanghai Generay Biotech Co., Ltd., China) into the pBMN-GFP vector (The Phoenix ${ }^{\mathrm{Tm}}$ retroviral system, Allele Biotech, USA) using the BamH I and EcoR I endonucleases (TaKaRa, Dalian, China) and was verified by DNA sequencing (Sangon, China). For transfection, the cells were seeded in 6-well plates at a density of $1 \times 104$ cells in $2 \mathrm{~mL}$ of culture medium per well and were allowed to attach for $24 \mathrm{~h}$. The cells were then transfected with either the CDX2 expression plasmid or a lentiviral vector control plasmid according to the manufacturer's protocol. To evaluate the role of the PI3K pathway in CDX2 regulation and trophoblast invasion, the CDX2-overexpressing cells were treated with LY294002 (Sigma, USA), which is a highly selective inhibitor of the PI3K pathway. After treatment with 0.1 uM LY294002 for $24 \mathrm{~h}$, then harvested for further testing.

\section{In vitro cell invasion assay}

The invasive capacity of the HTR-8/SVneo cells was tested after transfection. The cell invasion assay was performed using a QCM ECMatrix 24-well kit (Chemicon, ECM550, USA) according to the manufacturer's instructions. The cells were resuspended in RPMI-1640 medium without FBS. They were then seeded into the upper compartment of the invasion chamber. The lower compartment was then immediately loaded with $600 \mu \mathrm{L}$ medium containing $10 \% \mathrm{FBS}$ as a chemoattractant. After $24 \mathrm{~h}$ of incubation at $37^{\circ} \mathrm{C}$ in a humidified atmosphere containing $5 \% \mathrm{CO}_{2}$, non-migrating cells in the top compartment were completely removed by a sterile cotton swab, while those cells that had invaded into the lower chambers were stained with $0.04 \%$ Trypan Blue solution, washed with distilled water, and dried at room temperature. The invading cells were eluted with $100 \mu \mathrm{L}$ of $10 \%$ acetic acid. The absorbances were then measured at $546 \mathrm{~nm}$ and $578 \mathrm{~nm}$ using a spectrophotometer (MK3, Thermo, USA). Each experiment was performed in triplicate.

\section{MTT assay for cell proliferation}

The proliferation capacity of the trophoblast cells was assessed using an MTT (Sigma, MO, USA) assay according to the manufacturer's instructions. The CDX2-overexpressing HRT-8/SVneo cells and the control cells were harvested with $0.05 \%$ trypsin-EDTA and were resuspended in medium. A total of $10^{4}$ cells in $100 \mu \mathrm{L}$ medium per well were seeded into 96 -well plates and incubated for $48 \mathrm{~h}$ at $37^{\circ} \mathrm{C}$ and $5 \% \mathrm{CO}_{2}$ in a humidified incubator. Twenty microliters of MTT ( $5 \mathrm{mg} / \mathrm{mL}$ stock) was then added to each well, and the plates were incubated for another $4 \mathrm{~h}$. The medium was then removed, and $150 \mu \mathrm{L}$ of dimethyl sulfoxide (DMSO) was added. The plates were gently mixed on a shaker at room temperature for $10 \mathrm{~min}$. Finally, the absorbances were measured at $490 \mathrm{~nm}$ using a spectrophotometer. There were five technical replicates per sample in each experiment, and each experiment was independently repeated three times.

\section{RNA extraction and quantitative real-time PCR}

TRIzol (Invitrogen, USA) was used to extract total RNA from approximately $5 \times 10^{6}$ cells. Following extraction, $1 \mu \mathrm{g}$ of RNA was reverse transcribed using Superscript II reverse transcriptase (Invitrogen, USA) and a random primer according to the manufacturer's instructions. Quantitative real-time PCR reactions were performed using SYBR Green (Applied Biosystems, USA) on an ABI 7500 system. Melting curve analyses were performed to evaluate the primer specificities. The PCR primers are listed in Table 1. The results were normalized to the level of $18 \mathrm{~S}$ rRNA. There were three technical replicates and at least three biological replicates per sample.

\section{Western blotting}

Protein extracts were prepared from transfected HTR-8/SVneo cells using RIPA lysis buffer (Sigma, USA). Protein concentration was measured using the bicinchoninic acid (BCA) method. Samples containing equal amounts of proteins $(100 \mu \mathrm{g})$ were separated by $10 \%$ sodium dodecyl sulfate polyacrylamide gel electrophoresis (SDS-PAGE) and were transferred to polyvinylidene difluoride (PVDF) membranes. The membranes were blocked with $5 \%$ nonfat dry milk in a Tris-buffer containing $0.05 \%$ Tween-20 (TBST) for $1 \mathrm{~h}$ and were then incubated with the primary antibodies at 1:1000 dilution. The membranes were then 
Table 1. The primer sequences used for the quantitative real-time PCR (F: forward primer and R: reverse primer)

\begin{tabular}{|c|c|c|}
\hline Gene & Primer sequence & Product size (bp) \\
\hline \multirow[b]{2}{*}{$\mathrm{Cdx} 2$} & F : 5'-GTCCCTCGGCAGCCAAGTGAAAAC-3' & \multirow[b]{2}{*}{120} \\
\hline & R: 5'-TCCGGATGGTGATGTAGCGACTGTAG-3' & \\
\hline \multirow[b]{2}{*}{ MMP-9 } & F : 5'-TGCCCGGACCAAGGATACAGTTT-3' & \multirow[b]{2}{*}{184} \\
\hline & R : 5'-GTTCAGGGCGAGGACCATAGAGG-3' & \\
\hline \multirow[b]{2}{*}{ TIMP-1 } & F: 5'-ACCGCAGCGAGGAGTTTCT-3' & \multirow[b]{2}{*}{185} \\
\hline & R : 5'-CAGTTTGCAGGGGATGGATAA-3' & \\
\hline \multirow[b]{2}{*}{ PI3K } & F : 5'-ATGGGGATGATTTACGGCAAGATA-3' & \multirow[b]{2}{*}{151} \\
\hline & R : 5'-TTTCGCACCACCTCAATAAGTC-3' & \\
\hline \multirow[b]{2}{*}{ AKT } & F: 5'-CCCCCGAGGTGCTGGAGGACAAT-3' & \multirow[b]{2}{*}{97} \\
\hline & R: 5'-AAGGGCAGGCGACCGCACATCAT-3 & \\
\hline \multirow[t]{2}{*}{$18 \mathrm{~S}$} & F : 5'-AGTCGCCGTGCCTACCAT-3' & \multirow{2}{*}{129} \\
\hline & R: 5'-CGGGTCGGGAGTGGGTAAT-3' & \\
\hline
\end{tabular}

incubated with HRP-conjugated goat anti-rabbit IgG. Chemiluminescence detection and quantification of protein bands were performed as described previously [27]. Densitometry analysis of relative protein levels was performed using the Image J software $[28,29]$. All experiments were repeated at least three times.

\section{Statistical analysis}

We used the SPSS 20.0 statistical software for data analysis. The data are expressed as the means \pm standard deviations (SD). Where appropriate, statistical analysis were performed with analysis of variance (ANOVA) test. Otherwise, comparisons between groups were conducted using Student's $t$ test. $\mathrm{P}<0.05$ was considered statistically significant.

\section{Results}

Overexpression of CDX2 in HTR-8/SVneo cells

The HTR-8/SVneo cells were seeded into 6-well plates and transfected with either the CDX2 expression plasmid or the control plasmid. After $48 \mathrm{~h}$, the cells were harvested for RNA and protein extraction. The protein expression of CDX2 was verified by western blotting (Fig. 1A). The mRNA expression of CDX2 was assessed by quantitative real-time PCR, and the level was 5.1-fold higher (Fig. 1B, Student's $t$ test, ${ }^{* *} \mathrm{p}<0.01$ ) in the CDX2-overexpressing cells than in the control.

\section{Effects of CDX2 overexpression on trophoblast invasion and proliferation}

We transiently overexpressed CDX2 in the HTR-8/SVneo cells to study the roles of CDX2 in trophoblast invasion and proliferation using the assays described above. We found that the invasion capacity of the CDX2-overexpressing cells was significantly higher than that of the control. The absorbances of the invaded cells were measured at $546 \mathrm{~nm}$ and 578 $\mathrm{nm}$. The data are presented as means $\pm \mathrm{SD}$, and the difference between the two groups of samples was statistically significant at $\mathrm{p}<0.05$ (Student's $t$ test, Fig. 2A, 2B). An MTT assay was performed to determine whether the increase in invasion by the CDX2-overexpressing 
Fig. 1. $\mathrm{CDX} 2$ overexpression in HTR-8/SVneo cells. HTR-8/SVneo trophoblast cells were transfected with pBMN-CDX2-GFP (CDX2) or the empty vector pBMN-GFP (Control). (A) Western blot analysis of CDX2 expression in the CDX2-overexpressing HTR-8/SVneo cells and the control cells. $\beta$-Actin was used as an internal control. (B) The relative mRNA expression level of CDX2 was measured by quantitative real-time PCR. Data are represented as means \pm SD. Student's $t$ test, ${ }^{* *} \mathrm{p}<0.01 \mathrm{com}-$ pared with the control.

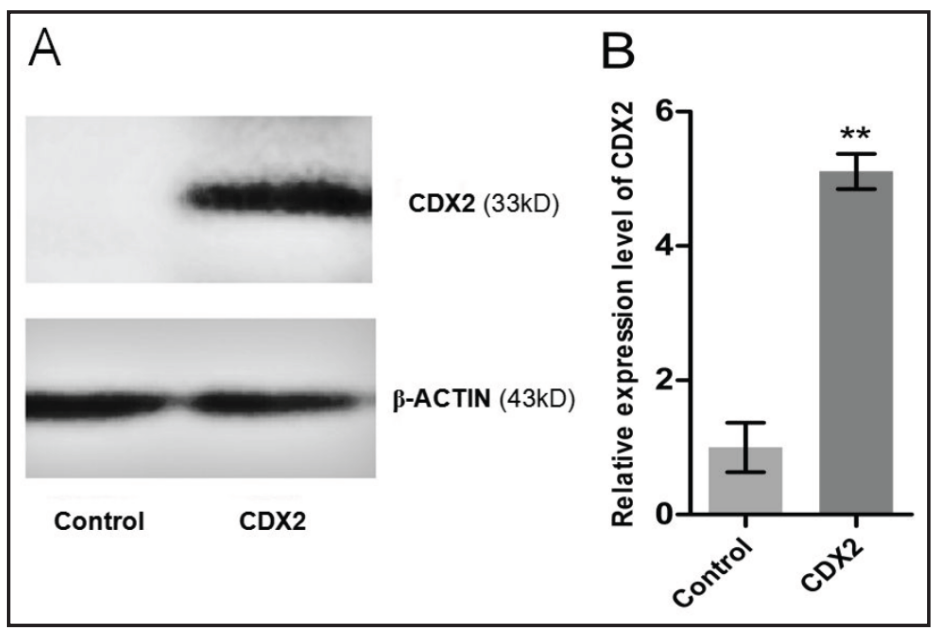

B
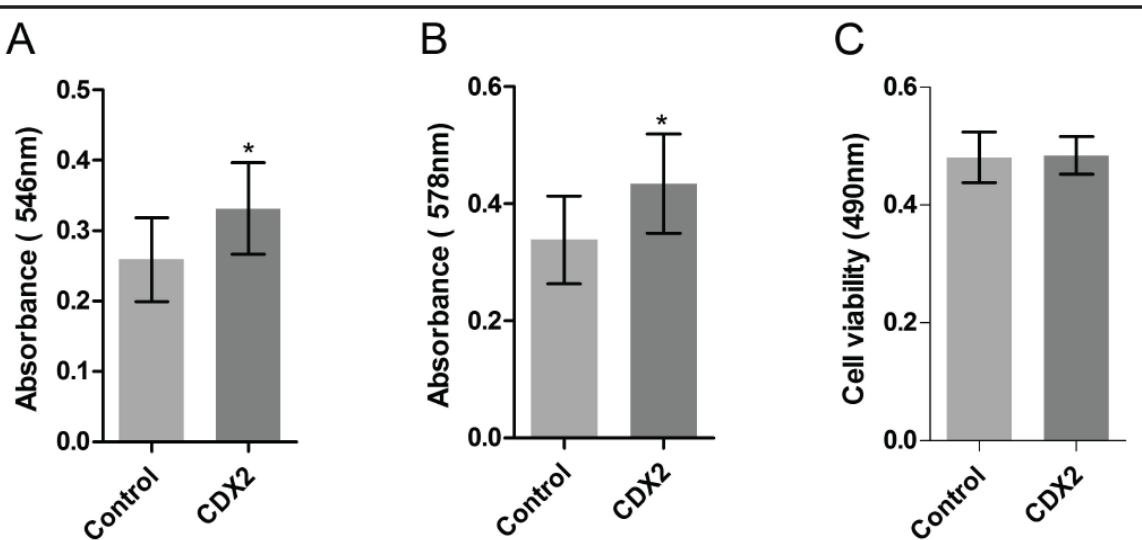

Fig. 2. Effects of CDX2 expression on HTR-8/SVneo cell invasion and proliferation. (A and B) Overexpression of CDX2 enhanced trophoblast invasion. The absorbances of invaded cells were measured at $546 \mathrm{~nm}$ and $578 \mathrm{~nm}$. Data are represented as means \pm SD; Student's $t$ test, ${ }^{*} \mathrm{p}<0.05$ compared with the control. (C) Cell proliferation measurements revealed no significant difference between the two groups (Student's $t$ test, p>0.05). Three independent experiments produced similar results.

cells was caused by increased cell proliferation. The results indicated that the proliferation capacity of the cells was not significantly changed when CDX2 was overexpressed (Fig. 2C). Three independent experiments produced similar results.

\section{The effects of CDX2 on MMP-9 and TIMP-1 expression}

We measured the protein expression levels of MMP-9 and TIMP-1 by western blotting. Overexpressing CDX2 significantly increased MMP-9 expression and decreased TIMP-1 (the inhibitor of MMP-9) expression in the HTR-8/SVneo cells (Fig. 3A). Densitometry analysis revealed that MMP-9 was increased to $340 \%$ compared with the control, whereas TIMP-1 was decreased to $35 \%$ relative to the control (Fig. 3B). Additionally, quantitative real-time PCR analysis showed the same trend as the western blots, with MMP-9 mRNA up-regulated and TIMP-1 mRNA down-regulated in CDX2-overexpressing HTR-8/SVneo cells (Fig. 3C).

\section{Regulation of CDX2 expression via the PI3K/AKT pathway}

To elucidate the mechanisms through which CDX2 promotes trophoblast invasion, we evaluated PI3K/AKT signaling in trophoblast cells. We measured PI3K and p-AKT expression by western blotting and quantitative real-time PCR in the CDX2-overexpressing HTR-8/ 


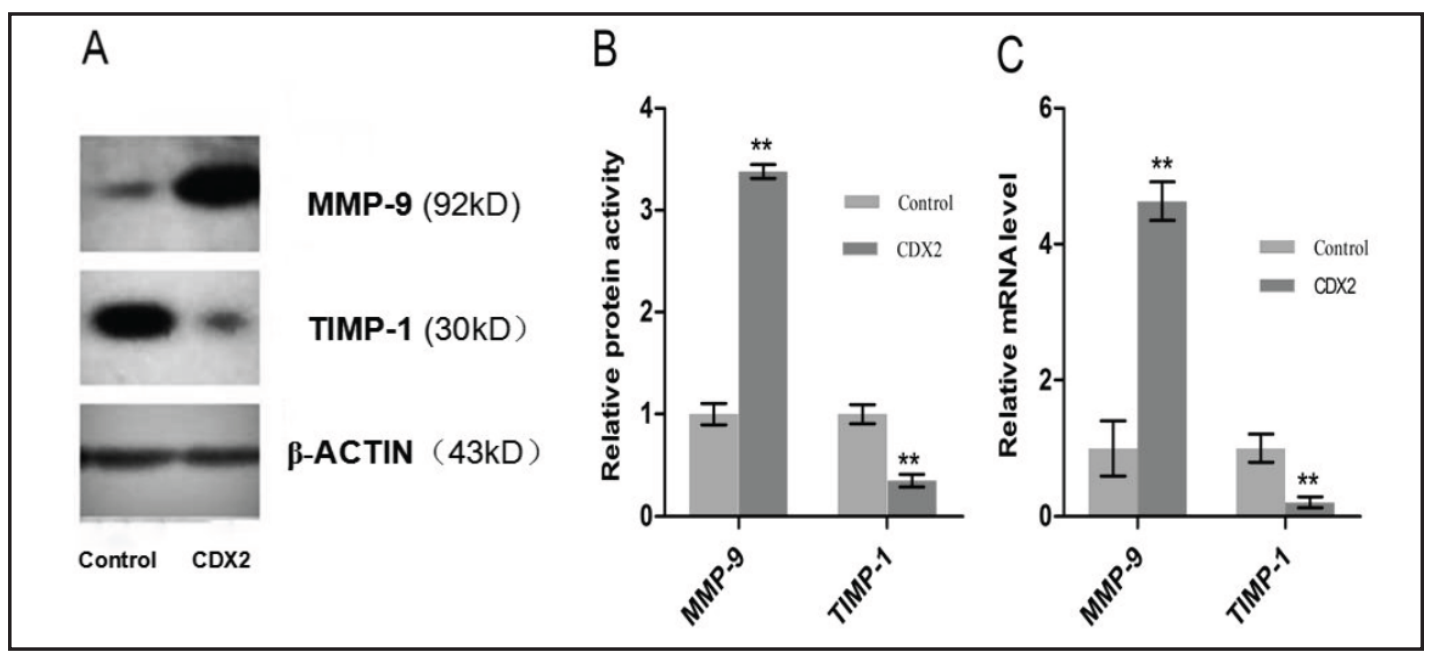

Fig. 3. Effects of CDX2 on MMP-9 and TIMP-1 expression. (A) MMP-9 and TIMP-1 protein expression was assessed by western blots in HTR-8/SVneo trophoblast cells transfected with pBMN-GFP (control) or pBMNCDX2-GFP (CDX2). (B) MMP-9 and TIMP-1 protein expression in control and CDX2-overexpressing HTR-8/ SVneo trophoblasts. Three independent experiments produced similar results. Data are represented as means \pm SD. ANOVA-test, ${ }^{* *} \mathrm{p}<0.01$ compared with the control. (C) Quantitative real-time PCR analysis of MMP9 and TIMP-1 mRNA expression in CDX2-overexpressing HTR-8/SVneo cells and the control cells (ANOVAtest, ${ }^{* *} \mathrm{p}<0.01$ ). Data are represented as means \pm SD from three independent experiments.

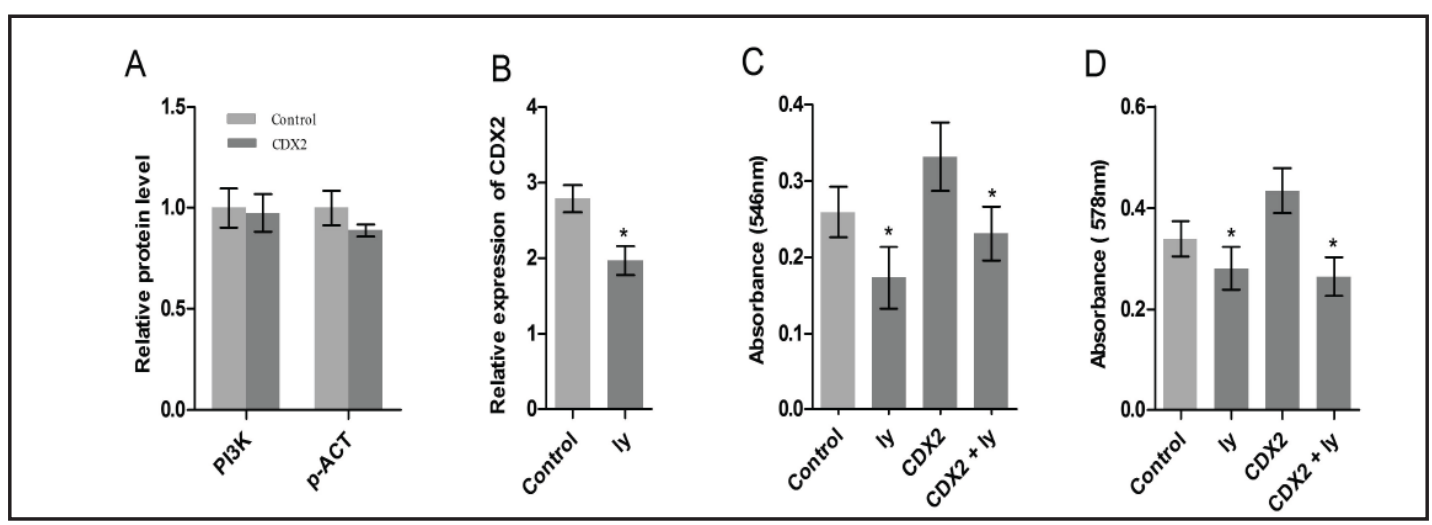

Fig. 4. Regulation of CDX2 expression via the PI3K/AKT pathway in HTR-8/SVneo cells. (A) PI3K and pAKT protein expression in control and CDX2-overexpressing HTR-8/SVneo trophoblasts. No significant difference was detected between the two groups. (B) Inhibition of PI3K signaling by LY294002 (ly) decreased CDX2 mRNA expression in HTR-8/SVneo trophoblast cells. (C and D) Effect of LY294002 on the invasion capacity of CDX2-overexpressing HTR-8/SVneo trophoblast cells. Data are represented as means \pm SD from three independent experiments (ANOVA-test, ${ }^{*} \mathrm{p}<0.05$ ).

SVneo cells and the control cells. Surprisingly, CDX2 did not affect PI3K/AKT signaling. No significant difference was observed for PI3K or p-AKT at either the mRNA or the protein level (Fig. 4A). However, when PI3K signaling was blocked by the specific inhibitor LY294002 in the HTR-8/SVneo cells, CDX2 expression was reduced (Fig. 4B) and the invasion capacity of the trophoblasts was decreased compared with the control cells (Fig. 4C, Fig. 4D: $0.173 \pm 0.041$ / $0.281 \pm 0.042$ vs. $0.259 \pm 0.033 / 0.339 \pm 0.035 p<0.05$ ). Invasion was also reduced when PI3K signaling was inhibited by LY294002 in the CDX2-overexpressing cells (Fig. 4C, Fig. 4D: $0.231 \pm 0.035 / 0.264 \pm 0.038$ vs. $0.322 \pm 0.045 / 0.435 \pm 0.045, \mathrm{p}<0.05)$. The absorbances of the invaded cells were measured at $546 \mathrm{~nm}$ and $578 \mathrm{~nm}$ using a spectrophotometer (Fig. 4C, 4D). Taken together, these data suggested that PI3K/AKT signaling might regulate CDX2 
expression in HTR-8/SVneo cells; however, CDX2 does not appear to reciprocally regulate the PI3K/AKT pathway.

\section{Discussion}

Like tumor cells, invasiveness is a feature of trophoblasts; however, unlike tumor invasion, trophoblast invasion is a strictly controlled physiological event. The placenta is a critical organ for the development of the fetus in utero. Trophoblast invasion into the uterine spiral arteries and the rebuilding of the arteries are crucial for normal placental development $[2,3,30]$. Imperfect uterine spiral artery rebuilding and inadequate trophoblast invasion have been associated with a range of pregnancy-related diseases [5-9].

CDX2 is a member of the caudal-related homeobox gene family and is involved in differentiation, proliferation, and maintenance of intestinal epithelial cells [19-22]. Using intestinal cell lines, numerous studies have indicated that CDX2 acts as a tumor suppressor by inhibiting cell proliferation [21, 22]. However, it has also been reported that CDX2 is associated with trophectoderm differentiation [24, 25, 31]. Our previous study found that CDX2 is hypomethylated in human severe pre-eclampsia placental tissues. Therefore, we sought to further investigate the effect of CDX2 on trophoblast invasion and proliferation. In this study, we found that CDX2 overexpression increased the invasiveness of the HTR-8/ SVneo cells (Fig. 2A, 2B) but did not affect cell proliferation (Fig. 2C). CDX2 may function in trophoblast invasion to prevent shallow placenta implantation and other pregnancy-related diseases.

At present, it is known that several cytokines and growth factors are associated with the regulation of trophoblast invasion [10-15, 32, 33]. However, very few of these regulatory factors have been investigated in depth. Recent reports have demonstrated that MMP-9 downregulation and TIMP-1 up-regulation can both lead to reduced trophoblast invasiveness [15-17]. We have also investigated the roles of MMP-9 and TIMP-1 in trophoblast invasion. Previous reports have shown that CDX2 inhibits invasion of Lovo cells by reducing expression of MMP-2 [34] and CDX1 restricts the invasion of HTR-8/SVneo trophoblast cells by inhibiting MMP-9 expression [35]. So we investage the effects of CDX2 on MMP-9 and TIMP1 expression. Our present study shows that CDX2 overexpression significantly promotes MMP-9 expression and inhibits TIMP-1 expression in HTR-8/SVneo trophoblast cells (Fig. 3B, 3C). These data suggest that CDX2 enhances trophoblast invasion by stimulating MMP-9 secretion while inhibiting TIMP-1 expression.

The PI3K/AKT signaling pathway plays important roles in cell growth, proliferation, migration and invasion [27, 36]. This pathway is activated in pre-eclamptic placentas to regulate cell proliferation [37]. Previous reports have shown that PI3K and AKT play an important role in regulating trophoblast invasion through the upregulation of MMP-9/TIMP1 expression by EGF [38]. Literature also suggests that HCG stimulates trophoblast invasion through PI3K and AKT signaling [39]. Recent studies have shown that CDX-2 was a target of PI3K signaling [40, 41]. We investigated the effect of PI3K/Akt signaling on CDX2 expression in HTR8/SVneo cells. Inhibition of PI3K signaling with the specific inhibitor LY294002 decreased CDX2 expression (Fig. 4B). In contrast, CDX2 overexpression did not affect PI3K expression (Fig. 4A). These data suggest that the PI3K/AKT pathway could regulate CDX2 expression.

In summary, our results demonstrated that CDX2 overexpression in HTR-8/SVneo cells enhanced cell invasion by promoting MMP-9 expression and inhibiting TIMP-1 expression. Therefore, the expression level of CDX2 may have a significant impact on trophoblast invasion and may be associated with pregnancy-related diseases. Our findings may reveal new insights into the regulatory mechanisms of trophoblast invasion and may be an important contributor to the pathogenesis of pregnancy-related diseases. 
Jia et al.: CDX2 Enhances HTR-8/SVneo Cell Invasion through MMP Pathway

\section{Acknowledgments}

This study was supported by grants from the National Natural Science Foundation of China (No. 81200442), the Natural Science Foundation of Jiangsu Province, China (No. BK2011106), the Scientific and Technological Personnel Start Projects Foundation of Nanjing Health Bureau, Jiangsu Province, China (No. QYK11141), and the Selected Member of Nanjing Health Youth Talent Training Project (QRX11209).

\section{References}

1 Chen JZJ, Sheehan PM, Brennecke SP, Keogh RJ: Vessel remodeling, pregnancy hormones and extravillous trophoblast function. Mol Cell Endocrinol 2012;349:138-144.

- Hunkapiller NM, Fisher SJ: Placental remodeling of the uterine vasculature. Method Enzymol 2008;445:281-302.

3 Knöfler M, Pollheimer J: IFPA Award in Placentology lecture: molecular regulation of human trophoblast invasion. Placenta 2012;33:S55-S62.

4 Norwitz ER: Defective implantation and placentation: laying the blueprint for pregnancy complications. Reprod Biomed Online 2006;13:591-599.

5 Hiden U, Bilban M, Knöfler M, Desoye G: Kisspeptins and the placenta: regulation of trophoblast invasion. Rev Endocr Metab Dis 2007;8:31-39.

6 Ball E, Bulmer JN, Ayis S, Lyall F, Robson SC: Late sporadic miscarriage is associated with abnormalities in spiral artery transformation and trophoblast invasion. AM J Pathol 2006;208:535-542.

7 Goldman-Wohl D, Yagel S: Regulation of trophoblast invasion: from normal implantation to pre-eclampsia. Mol Cell Endocrinol 2002;187:233-238.

-8 Plaks V, Rinkenberger J, Dai J, Flannery M, Sund M, Kanasaki K, Werb, Z: Matrix metalloproteinase-9 deficiency phenocopies features of preeclampsia and intrauterine growth restriction. P Natl Acad Sci USA 2013;110:11109-11114.

9 Wells M: The pathology of gestational trophoblastic disease: recent advances. Pathology 2007;39:88-96.

10 Cui Y, Wang W, Dong N, Lou J, Srinivasan DK, Cheng W, Wu, Q: Role of corin in trophoblast invasion and uterine spiral artery remodelling in pregnancy. Nature 2012;484:246-250.

11 Shih IM, Hsu MY, Oldt III RJ, Herlyn M, Gearhart JD, Kurman RJ: The role of E-cadherin in the motility and invasion of implantation site intermediate trophoblast. Placenta 2002;23:706-715.

12 Huber AV, Saleh L, Bauer S, Husslein P, Knöfler M: TNF $\alpha$-mediated induction of PAI-1 restricts invasion of HTR-8/SVneo trophoblast cells. Placenta 2006;27:127-136.

13 Staun-Ram E, Goldman S, Gabarin D, Shalev E: Expression and importance of matrix metalloproteinase 2 and 9 (MMP-2 and-9) in human trophoblast invasion. Reprod Biol Endocrin 2004;2:59.

14 [14] Karmakar S, Das C: Regulation of Trophoblast Invasion by IL-1 $\beta$ and TGF- $\beta 1$. Am J Reprod Immunol 2002; 48: 210-219.

-15 Luo J, Qiao F, Yin X: Hypoxia induces FGF2 production by vascular endothelial cells and alters MMP9 and TIMP1 expression in extravillous trophoblasts and their invasiveness in a cocultured model. J Reprod Develop 2011;57:84-91.

16 Singh M, Kindelberger D, Nagymanyoki Z, Ng SW, Quick CM, Elias KM, Berkowitz, RS: Matrix metalloproteinases and their inhibitors and inducer in gestational trophoblastic diseases and normal placenta. Gynecol Oncol 2011;122:178-182.

17 Karthikeyan VJ, Lane DA, Beevers DG, Lip GYH, Blann AD: Matrix metalloproteinases and their tissue inhibitors in hypertension-related pregnancy complications. J Hum Hypertens 2013;27:72-78.

18 Zhu JY, Pang ZJ, Yu Y: Regulation of trophoblast invasion: the role of matrix metalloproteinases. Reviews in obstetrics and gynecology 2012;5:e137.

19 Beck F, Stringer EJ: The role of Cdx genes in the gut and in axial development. Biochem Soc T 2010;38:353357.

20 Silberg DG, Swain GP, Suh ER, Traber PG: Cdx1 and cdx2 expression during intestinal development. Gastroenterology 2000;119:961-971.

-21 Eda A, Osawa H, Yanaka I, Satoh K, Mutoh H, Kihira K: Expression of homeobox gene CDX2 precedes that of CDX1 during the progression of intestinal metaplasia. J Gastroentero 2002;37:94-100. 


\section{Cellular Physiology Cell Physiol Biochem 2014;34:628-636 \begin{tabular}{ll|l} 
Dol: 10.1159/000363028 & O 2014 S. Karger AG, Basel \\
www.karger.com/cpb
\end{tabular} \\ Jia et al.: CDX2 Enhances HTR-8/SVneo Cell Invasion through MMP Pathway}

22 Park SY, Jeong MS, Yoo M, Jang SB: Caudal-related homeodomain proteins CDX1/2 bind to DNA replicationrelated element binding factor. BBA-Proteins Proteom 2011;1814:1891-1899.

23 Jia, RZ, Zhang X, Hu P, Liu XM, Hua XD, Wang X, Ding HJ: Screening for differential methylation status in human placenta in preeclampsia using a CpG island plus promoter microarray. Int J Mol Med 2012;30:133.

-24 Tolkunova E, Cavaleri F, Eckardt S, Reinbold R, Christenson LK, Schöler HR, Tomilin, A: The CaudalRelated Protein Cdx2 Promotes Trophoblast Differentiation of Mouse Embryonic Stem Cells. Stem Cells 2006;24:139-144.

25 Murohashi M, Nakamura T, Tanaka S, Ichise T, Yoshida N, Yamamoto T, Gotoh, N: An FGF4-FRS2 $\alpha$-Cdx2 Axis in Trophoblast Stem Cells Induces Bmp4 to Regulate Proper Growth of Early Mouse Embryos. Stem Cells 2010;28:113-121.

-26 Suman P, Gupta SK: Comparative analysis of the invasion-associated genes expression pattern in first trimester trophoblastic (HTR-8/SVneo) and JEG-3 choriocarcinoma cells. Placenta 2012;3:874-877.

27 Wu WL, Gan WH, Tong ML, Li XL, Dai JZ, Zhang CM, Guo, XR: Over-expression of NYGGF4 (PID1) inhibits glucose transport in skeletal myotubes by blocking the IRS1/PI3K/AKT insulin pathway. Mol Genet Metab 2011;102:374-377.

28 Lee G, Papapetrou EP, Kim H, Chambers SM, Tomishima MJ, Fasano CA, Studer L: Modelling pathogenesis and treatment of familial dysautonomia using patient-specific iPSCs. Nature 2009;461:402-406.

29 Nakagawa I, Amano A, Mizushima N, Yamamoto A, Yamaguchi H, Kamimoto T, Yoshimori T: Autophagy defends cells against invading group A Streptococcus. Science 2004;306:1037-1040.

-30 Ren L, Liu YQ, Zhou WH, Zhang YZ: Trophoblast-derived chemokine CXCL12 promotes CXCR4 expression and invasion of human first-trimester decidual stromal cells. Hum Reprod 2012;27:366-374.

31 Thompson, Megan Elizabeth: Epigenetic Control of Gene Expression in the Placental Trophoblast (Doctoral dissertation). 2012.

-32 Lala PK, Chakraborty C: Factors regulating trophoblast migration and invasiveness: possible derangements contributing to pre-eclampsia and fetal injury. Placenta 2003;24:575-587.

-33 Bischoff P, Meisser A, Campana A: Paracrine and autocrine regulators of trophoblast invasion-a review. Placenta 2000; 1:S55-S60.

-34 Zheng JB, Sun XJ, QiJ, Li SS, Wang W, Ren HL, Du JK: Effects of homeodomain protein CDX2 expression on the proliferation and migration of lovo colon cancer cells. Pathology \& Oncology Research 2011;17:743751.

-35 Jia RZ, Rui C, Li JY, Cui XW, Wang X: CDX1 restricts the invasion of HTR-8/SVneo trophoblast cells by inhibiting MMP-9 expression. Placenta 2014;35:450-454.

-36 Duan C, Bauchat JR, Hsieh T: Phosphatidylinositol 3-kinase is required for insulin-like growth factor-1induced vascular smooth muscle cell proliferation and migration. Circ Res 2000;86:15-23.

37 Park JK, Jeong JW, Kang MY, Baek JC, Shin JK, Lee SA, Paik, WY: Inhibition of the PI3K-Akt Pathway Suppresses sFlt1 Expression in Human Placental Hypoxia Models In Vitro. Placenta 2010;31:621-629.

-38 Qiu Q, Yang M, Tsang BK, Gruslin A: EGF-induced trophoblast secretion of MMP-9 and TIMP-1 involves activation of both PI3K and MAPK signalling pathways. Reproduction 2004;128:355-363.

-39 Prast J, Saleh L, Husslein H., Sonderegger S, Helmer H, Knofler M: Human chorionic gonadotropin stimulates trophoblast invasion through extracellularly regulated kinase and AKT signaling. Endocrinology 2008;149:979-987.

-40 Bai ZG, Ye YJ, Shen DH, Lu YY, Zhang ZT, Wang S: PTEN expression and suppression of proliferation are associated with Cdx2 overexpression in gastric cancer cells. International journal of oncology 2013;42:1682-1691.

41 Kim S1, Domon-Dell C, Wang Q Chung DH, Di Cristofano A, Pandolfi PP, Freund JN, Evers BM: PTEN and TNF-alpha regulation of the intestinal-specific Cdx-2 homeobox gene through a PI3K, PKB/Akt, and NFkappaB-dependent pathway. Gastroenterology 2002;123:1163-1178. 